A multitude of treatments have been advocated for the management of tendon

\title{
Harnessing the stem cell for the treatment of tendon injuries: heralding a new dawn?
}

\section{R K W Smith, P M Webbon}

Stem cell technology may be useful in the treatment of tendon and ligament injuries

$\mathrm{S}$ train induced tendon or ligament injuries are an all too common consequence of athletic endeavour in both horses ${ }^{1}$ and humans, ${ }^{2-4}$ resulting in high morbidity and often compromising a return to the same level of activity.

In the horse, the most commonly injured structures are the weight bearing digital flexor tendons which lie on the palmar aspect of the metacarpus (fig 1). There are many similarities between the weight bearing tendons of the horse and the human athlete-for example, Achilles tendon-in function, matrix composition, and the nature of the injuries sustained. In contrast with positional tendons, such as the hand tendons, all weight bearing tendons function as springs, storing energy under weight bearing load for efficient locomotion. ${ }^{5}$ The horse has maximised this potential, resulting in efficiency of locomotion in excess of $100 \%$ at the gallop.

Whereas immature tendon shows an ability to adapt to the loads placed on it, this appears to be lost, or dramatically reduced, in the adult. ${ }^{78}$ Thus over-strain injuries, although they occur spontaneously during exercise, are preceded by progressive degeneration of the tendon matrix brought about by cumulative loading cycles, accelerated by competitive sport. It is not surprising therefore that weight bearing tendons, which operate close to their functional limit, commonly fail in both horses and humans.

Clinical injury results in a variable disruption of the tendon matrix, which induces an inflammatory response. This response is often short lived. Very soon after the injury, fibroplasia is initiated resulting in the formation of scar tissue within the tendon. In the later stages of repair, inflammation is no longer an obvious clinical feature, giving rise to a debate whether the disease is a tendinitis or a tendinopathy. The latter logically encompasses all stages, but the absence of inflammation does not indicate that it was not present at the outset even though it is often missed clinically in cases of mild repetitive injury. Over a number of months, the immature scar tissue remodels, increasing not only in strength but also in stiffness, which reduces the efficiency of the tendon as a spring, compromising the performance of the horse (or human) and putting the tendon at risk of reinjury.

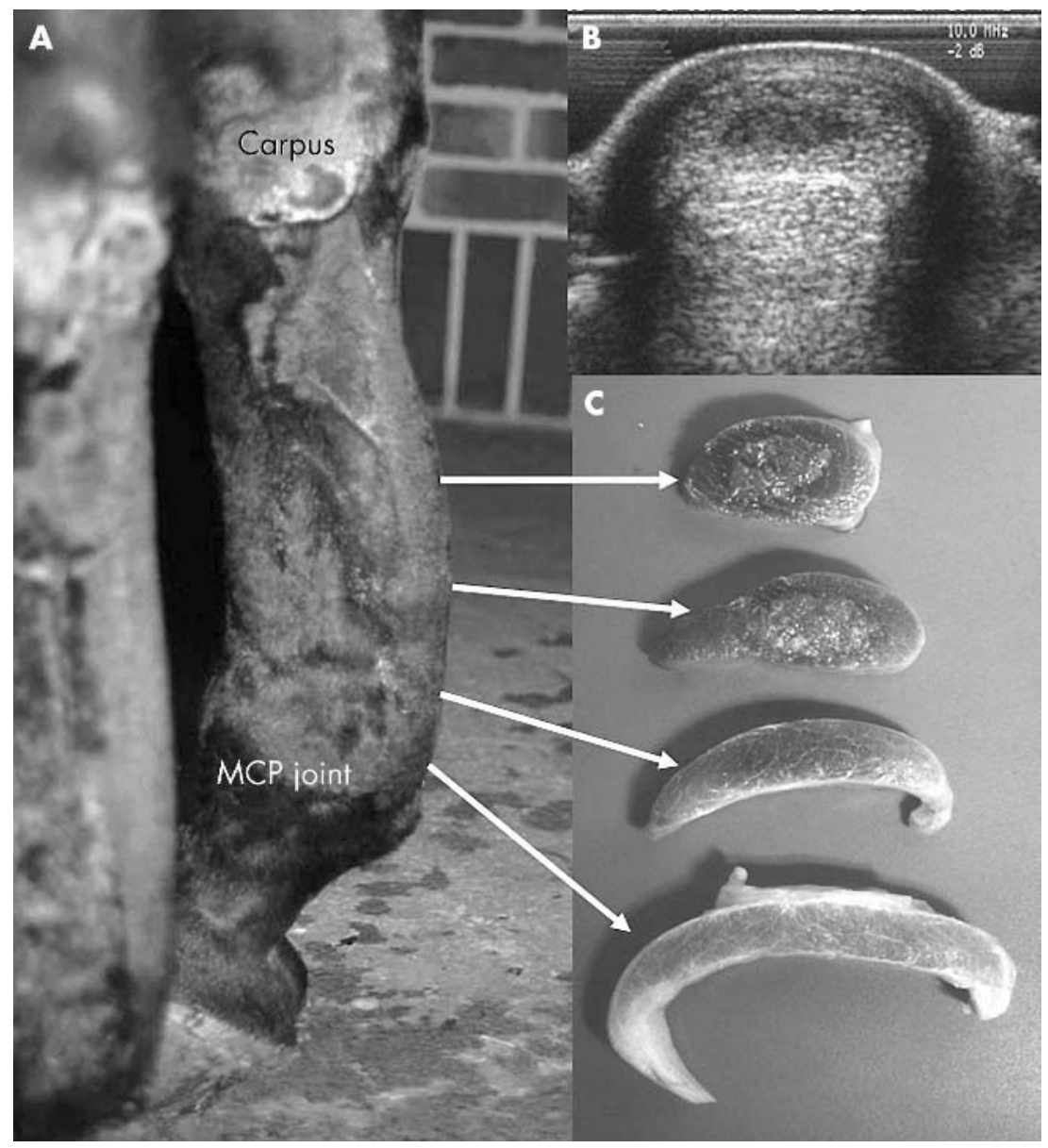

Figure 1 Equine superficial digital flexor tendinopathy. (A) The clinical appearance of the injury in the acute stages. The tendon, which lies on the most superficial aspect (immediately deep to the skin) of the palmar surface of the limb, becomes swollen, hot, and painful. This results in a curved, rather than the normally straight, contour to the back of the limb, hence, the colloquial term for the injury "a bowed leg". MCP, metacarpophalangeal. (B) A transverse ultrasonograph from the midmetacarpal region of a case similar to that shown in (A). Note the central hypoechoic region within the superficial digital flexor tendon characteristic of this injury. (C) Pathological sections of a corresponding injury, showing the preferential area of injury in the centre of the extra-thecal (midmetacarpal) region of the tendon. Interestingly the area of the tendon "impinged" on the back of the metacarpophalangeal joint where the tendon changes direction and is compressed is rarely injured. 
if we are going to be able to return equine and human athletes to full function.

\section{AUTOLOGOUS \\ TRANSPLANTATION OF MESENCHYMAL STEM CELLS INTO INJURED TENDONS: THE HOPE FOR THE FUTURE?}

At present, no treatment is successful at promoting the formation of tendon matrix. We have hypothesised that the best hope for engineering new tendon tissue that has compositional and functional similarities to non-degenerate weight bearing tendon will require a cell based approach with the delivery of mesenchymal stem cells, in far greater numbers than are present normally, into the damaged tendon.

Embryonic stem cells are truly pluripotential but have the disadvantages of being allogenic (although with immunological tolerance) and associated with a risk of teratoma formation. Postnatally derived stem cells are thought to be multipotential, having a restricted number of cell lineages into which they can differentiate. They are subdivided into haemopoietic (blood cell lines) and mesenchymal stem cells which can give rise to osteoblasts (bone), chondrocytes (cartilage), tenocytes (tendon and ligament), fibroblasts (scar tissue), adipocytes (fat), and myofibroblasts (myotubes). Furthermore, these stem cells can be recovered from adult tissue, and hence there is the possibility of autologous reimplantation.

"...the transplantation of mesenchymal stem cells into injured tendons has been shown to promote tendon healing in laboratory animal models."

Little work has been carried out into the tenocytic potential of mesenchymal stem cells and the growth factors and mediators required to elicit tenocyte differentiation, although a combination of mechanical stimuli and proximity to tenocytes and tendon matrix (as provided by direct implantation of cells into the tendon) are believed to be important. In spite of these unanswered questions, the transplantation of mesenchymal stem cells into injured tendons has been shown to promote tendon healing in laboratory animal models. Young et al ${ }^{9}$ seeded mesenchymal stem cells (so-called bone marrow derived stromal cells) on to a biodegradable scaffold in a $1 \mathrm{~cm}$ defect in a rabbit Achilles tendon. The results after 12 weeks showed the regeneration of new tendon-like tissue in the defect. Awad et $a l^{10}$ used a similar rabbit model, but investigated injury to the patellar tendon where mesenchymal stem cell mediated repair improved the biomechanical characteristics.

These experimental studies used laceration injuries, where maintaining the cells within the laceration site required a scaffold, which can also exert an influence, positively or negatively. However, equine digital flexor tendon strain injuries invariably provide a central core lesion which can retain implanted mesenchymal stem cells (fig 1). To take advantage of these experimental and clinical observations, we, in conjunction with VetCell Bioscience Ltd, have developed a stem cell based treatment for the management of acute tendon injuries in horses using autologous bone marrow derived stromal cells ${ }^{11}$ (summarised in fig 2).

Bone marrow is harvested from the sternum of the horse under standing sedation and shipped immediately to the laboratory, where the bone marrow stromal cells (or mesenchymal stem cells) are recovered and expanded in vitro until in excess of $4 \times 10^{6}$ cells are present. These cells are then suspended in citrated bone marrow supernatant for implantation under ultrasonographic guidance into the core lesion of the damaged tendon, again under standing sedation. The bone marrow supernatant is used to resuspend the stem cells to utilise its stimulatory effects ( $\mathrm{J}$ Smith, unpublished data), and citrate is the anticoagulant of choice as the injected solution clots after injection (because of diffusion of the citrate), thereby immobilising the implanted cells and reducing the risk of leakage from the needle injection sites.

Clinical experience has suggested that the optimal time for bone marrow aspiration is within the first month after injury so that implantation is performed one to two months after injury. This allows the initial inflammatory debridement phase to subside and a suitable granulation bed to form, which would support the survival of the implanted stem cells, but before substantial fibrosis had occurred. Mesenchymal stem cells can also be harvested before injury and/or stored for subsequent use if the animal suffers a reinjury or a different tendon/ligament injury. We have indeed been able to show the successful reculture and implantation of frozen, stored mesenchymal stem cells, without the need for recovery of further bone marrow.

In cases where the lesion is present within a tendon sheath, the implantation is performed under tenoscopic guidance under general anaesthesia to ensure no surface defects are present from which the implanted cells could leak into the tendon sheath. If no defect

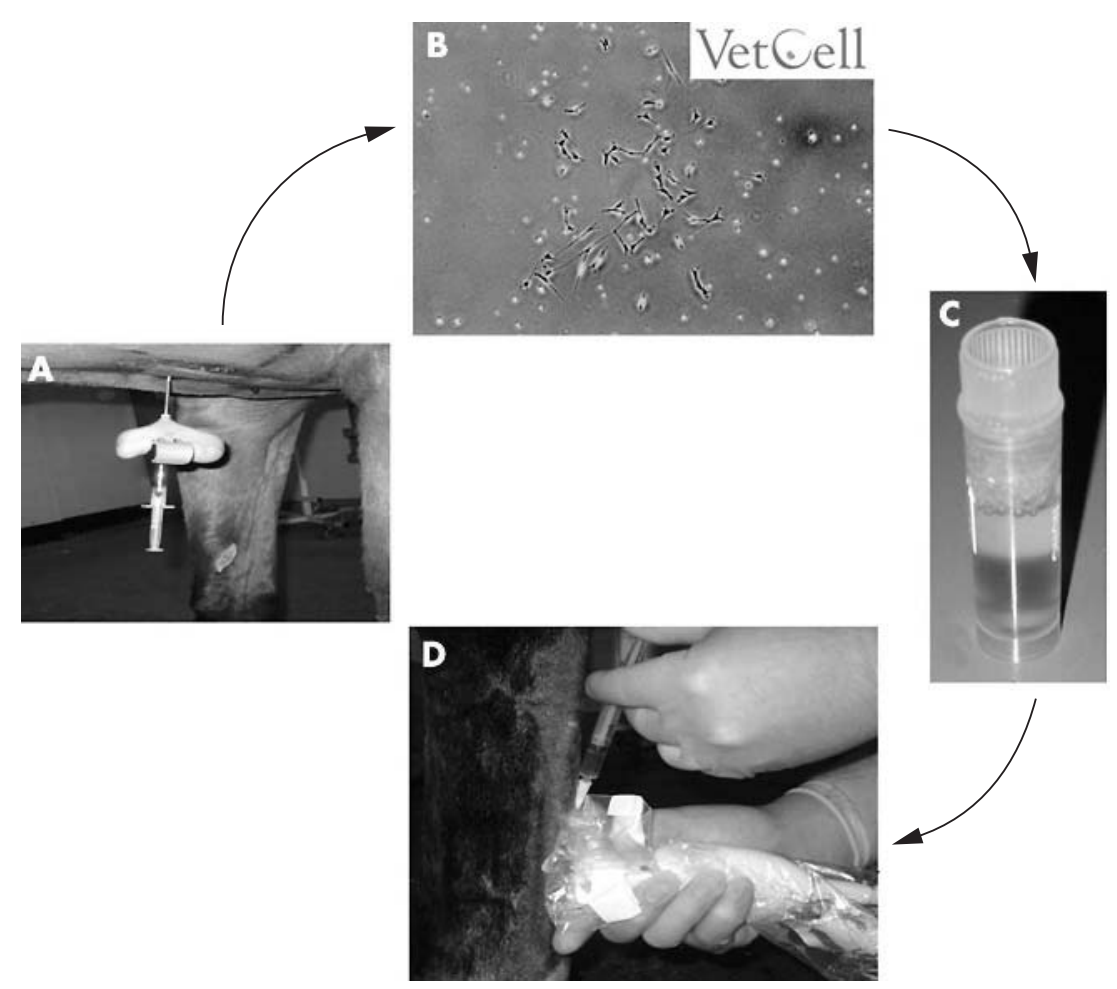

Figure 2 The stem cell based approach to the treatment of equine superficial digital flexor tendinopathy. (A) Aspiration of bone marrow from the sternum of the standing horse. (B) Recovery and expansion of mesenchymal stem cells (bone marrow stromal cells) by VetCell Bioscience Ltd. (C) Resuspension of $>4 \times 10^{6}$ stem cells in citrated bone marrow supernatant. (D) Sterile implantation of the cells into the central core lesion under ultrasonographically guided injection. 
A

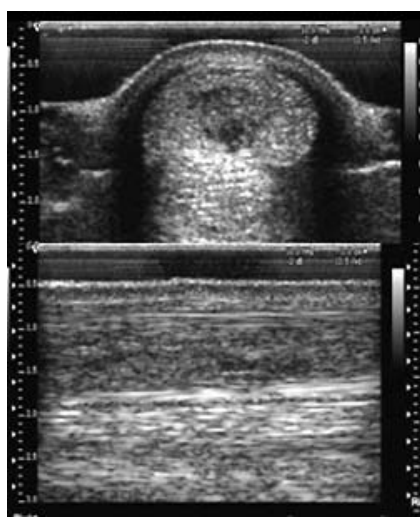

BM aspiration ( $t=0)$
B

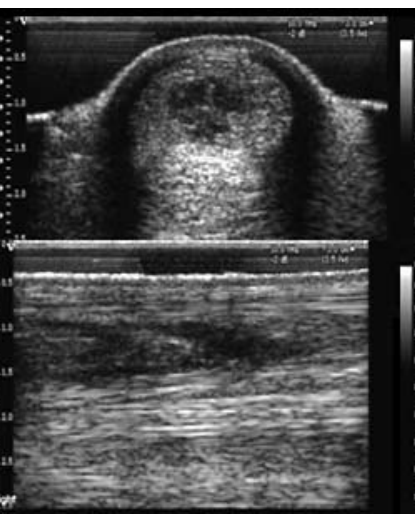

C

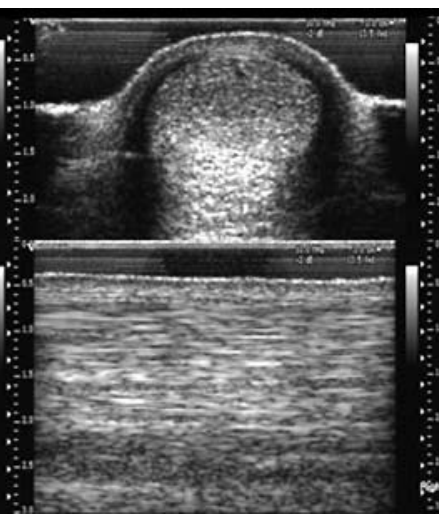

D

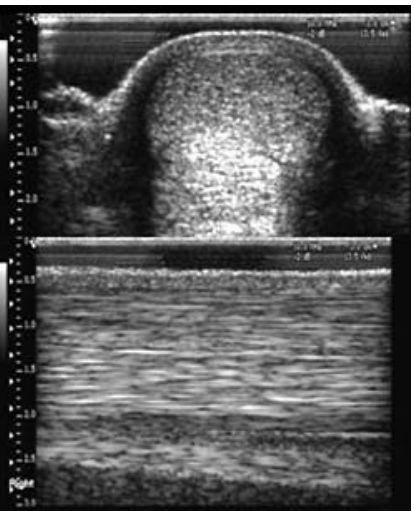

Pre-implantation (+1 month)

+2 months

Figure 3 Sequential transverse (top row) and longitudinal (bottom row) ultrasonographs taken from a horse treated by the stem cell technique. Note the rapid infilling of the lesion within one month of implantation, whereas there was little change in the lesion in the preceding month.

is present, the cells are then implanted accurately into the lesion under a combination of tenoscopic and ultrasonographic control. Direct tenoscopic visualisation of the injected area after withdrawal of the needle has also enabled us to show that there is no leakage of the implanted solution from the needle tract, confirming that the implanted solution stays within the tendon.

After implantation, the horses enter a controlled ascending exercise regimen with regular ultrasonographic examinations every two to three months, so that they are back in full training after 48 weeks. To date, more than 100 horses from the United Kingdom and across Europe have been treated using this technique. Ultrasonographic evaluation after implantation has revealed no deleterious effects to the healing tendon by the implantation, and shown evidence of rapid infilling of the defect (fig 3). Six cases were also evaluated radiographically and scintigraphically after three months and there was no evidence of any bone formation within the treated tendon.

At this stage it is not possible to show significantly improved healing over conventionally treated animals because of limited case numbers with sufficient follow up time. Furthermore equine superficial digital flexor tendon injuries are highly variable, and controlled studies are almost impossible to perform in an environment in which treatment is essentially private and dominated by owner/trainer preference. So far, a limited number of racehorses has been followed for over 12 months and have shown rates of return to racing that are better than those published in a previous study of racehorses with similar severity of injuries. ${ }^{12}$ Reinjuries have occurred, although these horses were implanted later when fibrosis was already advanced, suggesting that early implantation is important.

The technique has proved highly practical within the clinic environment. To avoid misuse of this technology, VetCell Bioscience Ltd runs training courses, and more than 200 veterinarians Europe-wide have attended. Bone marrow aspirates are submitted for stem cell recovery and cultured cells returned to the veterinarians for implantation in special couriered containers.

In summary, this technology has shown encouraging, but not yet proven, efficacy for treating acute tendon lesions in horses. A truly valid comparison between stem cell treated and conventionally managed cases will require a large clinical trial, but we believe that the technique at least deserves further evaluation for its use in a variety of tendon and ligament injuries. Should this technique be proven effective, it is hoped that our experience with horses will pave the way for this technology to be used successfully in human tendon and ligament injuries.

Br J Sports Med 2005;39:582-584. doi: 10.1136/bjsm.2005.015834

\section{Authors' affiliations}

R K W Smith, Department of Veterinary Clinical Sciences, The Royal Veterinary College, University of London, Hatfield, Herts AL9 7TA, UK

P M Webbon, The Jockey Club, 151

Shaftesbury Avenue, London WC2H 8AL, UK

Correspondence to: Professor Smith

Department of Veterinary Clinical Sciences, The
Royal Veterinary College, University of London Hatfield, Herts AL9 7TA, UK; rksmith@rvc.ac.uk Competing interests: RKWS is a director of VetCell Bioscience Ltd.

\section{REFERENCES}

1 Williams RB, Harkins LS, Hammond CJ, et al. Racehorse injuries, clinical problems and fatalities recorded on British racecourses from flat racing and National Hunt racing during 1996, 1997 and 1998. Equine Vet $J$ 2001;33:478-86.

2 Moller A, Astron M, Westlin N. Increasing incidence of Achilles tendon rupture. Acta Orthop Scand 1996:67:479-481.

3 Houshian S, Tscherning T, Riegels-Nielsen P. The epidemiology of Achilles tendon rupture in a Danish county. Injury 1998;29:651-4.

4 Maffulli N, Waterston SW, Squair J, et al. Changing incidence of Achilles tendon rupture in Scotland: a 15-year study. Clin J Sport Med 1999;9:157-60

5 Wilson AM, McGuigan MP, Su A, et al. Horses damp the spring in their step. Nature 2001:414:895-8.

6 Minetti AE, Ardigo LP, Reinach E, et al. The relationship between mechanical work and energy expenditure of locomotion in horses. J Exp Biol 1999.202.2329-38.

7 Smith RKW, Birch HL, Patterson-Kane J, et al. Should equine athletes commence training during skeletal development? Changes in tendon matrix associated with development, ageing, function and exercise. Equine Vet J 1999;(suppl 31): 201-9.

8 Smith RKW, Birch HL, Goodman S, et al. The influence of ageing and exercise on tendon growth and degeneration: hypotheses for the initiation and prevention of strain-induced tendinopathies. Comp Biochem Physiol A Mol Integr Physiol 2002; 133:1039-50.

9 Young RG, Butler DL, Weber W, et al. Use of mesenchymal stem cells in a collagen matrix for Achilles tendon repair. J Orthop Res 1998; 16:406-13

10 Awad HA, Butler DL, Boivin GP, et al. Autologous mesenchymal stem cell-mediated repair of tendon. Tissue Eng 1999;5:267-77.

11 Smith RKW, Korda M, Blunn GW, et al. Isolation and implantation of autologous equine mesenchymal stem cells from bone marrow into the superficial digital flexor tendon as a potentia novel treatment. Equine Vet J 2003:35:99-102.

12 Marr CM, Love S, Boyd JS, et al. Factors affecting the clinical outcome of injuries to the superficial digital flexor tendon in National Hunt and pointto-point racehorses. Vet $\operatorname{Rec} 1993 ; 132: 476-9$. 